Homology, Homotopy and Applications, vol.21(1), 2019, pp.323-340

\title{
GENERALIZED GOTTLIEB AND WHITEHEAD CENTER GROUPS OF SPACE FORMS
}

\author{
MAREK GOLASIŃSKI AND THIAGO DE MELO
}

(communicated by Donald M. Davis)

\begin{abstract}
We extend Oprea's result that the Gottlieb group $G_{1}\left(\mathbb{S}^{2 n+1} / H\right)$ is $\mathcal{Z} H$ (the center of $H$ ) and show that for a map $f: A \rightarrow$ $\mathbb{S}^{2 n+1} / H$, under some conditions on $A$, we have $G_{1}^{f}\left(\mathbb{S}^{2 n+1} / H\right)=$ $\mathcal{Z}_{H} f_{*}\left(\pi_{1}(A)\right)$, the centralizer of the image $f_{*}\left(\pi_{1}(A)\right)$ in $H$. Then, we compute or estimate the groups $G_{m}^{f}\left(\mathbb{S}^{2 n+1} / H\right)$ and $P_{m}^{f}\left(\mathbb{S}^{2 n+1} / H\right)$ for certain $m>1$.
\end{abstract}

\section{Introduction}

Throughout this paper, all spaces are path-connected with the homotopy types of $C W$-complexes and maps and homotopies are based. We use the standard terminology and notations from homotopy theory, mainly from [10] and [23]. We do not distinguish between a map and its homotopy class.

Let $X$ be a connected space and $\mathbb{S}^{m}$ the $m$-sphere. The $m^{\text {th }}$ Gottlieb group $G_{m}(X)$ of $X$, defined first for $m=1$ in [11] and then for $m \geqslant 1$ in [12], is the subgroup of the $m^{\text {th }}$ homotopy group $\pi_{m}(X)$ consisting of all elements which can be represented by a map $\alpha: \mathbb{S}^{m} \rightarrow X$ such that $\operatorname{id}_{X} \vee \alpha: X \vee \mathbb{S}^{m} \rightarrow X$ extends (up to homotopy) to a map $F: X \times \mathbb{S}^{m} \rightarrow X$. Following [12], we recall that $P_{m}(X)$ is the set of elements of $\pi_{m}(X)$ whose Whitehead products with all elements of all homotopy groups of $X$ are zero. It turns out that $P_{m}(X)$ forms a subgroup of $\pi_{m}(X)$ called the Whitehead center group and, by [12, Proposition 2.3], we also have $G_{m}(X) \subseteq P_{m}(X)$. Some advanced attempts to compute the groups $G_{m}(X)$ and $P_{m}(X)$ for spheres and projective spaces have been made in $[\mathbf{1 0}]$.

Now, given a map $f: A \rightarrow X$, in view of [12] (see also [19]), the $m^{\text {th }}$ generalized Gottlieb group $G_{m}^{f}(X)$ is defined as the subgroup of the $m^{\text {th }}$ homotopy group $\pi_{m}(X)$ consisting of all elements which can be represented by a map $\alpha: \mathbb{S}^{m} \rightarrow X$ such that $f \vee \alpha: A \vee \mathbb{S}^{m} \rightarrow X$ extends (up to homotopy) to a map $F: A \times \mathbb{S}^{m} \rightarrow X$. According to the literature, we say that the map $F$ is affiliated to $\alpha$. If $A=X$ then the group $G_{1}^{f}(X)$, also denoted by $J(f)$, is called the Jiang group of the map $f: X \rightarrow X$ in honor of Bo-Ju Jiang who recognized in [17] their importance to the Nielsen-Wecken

This work was supported by CAPES - Ciência sem Fronteiras, grant 88881.068125/2014-01.

Received June 1, 2018, revised September 6, 2018; published on October 31, 2018.

2010 Mathematics Subject Classification: 55Q52, 57S17, 55Q15, 55R05.

Key words and phrases: classifying space, Gottlieb group, homology group, homotopy group, MoorePostnikov tower, $n$-equivalence, projective space, space form, Whitehead center group, Whitehead product.

Article available at http://dx.doi.org/10.4310/HHA.2019.v21.n1.a15

Copyright (C) 2018, International Press. Permission to copy for private use granted. 
theory of fixed point classes. The role the group $J(f)$ played in that theory has been intensively studied in the book [7] as well.

The $m^{\text {th }}$ generalized Whitehead center group $P_{m}^{f}(X)$, as defined in [19], is the set of all elements $\alpha \in \pi_{m}(X)$ whose Whitehead products $[\alpha, f \beta]$ are zero for all $\beta \in \pi_{l}(A)$ with $l \geqslant 1$. It turns out that $P_{m}^{f}(X)$ forms a subgroup of $\pi_{m}(X)$ and $G_{m}^{f}(X) \subseteq P_{m}^{f}(X)$.

Given a free action $H \times \mathbb{S}^{2 n+1} \rightarrow \mathbb{S}^{2 n+1}$ of a finite group $H$ on $\mathbb{S}^{2 n+1}$, Oprea [20] has shown that $G_{1}\left(\mathbb{S}^{2 n+1} / H\right)=\mathcal{Z} H$, the center of $H$. Further, in the special case of a linear action of $H$ on $\mathbb{S}^{2 n+1}$, a very nice representation-theoretic proof of that fact has been given in [5]. In this paper, we generalize Oprea's results to the case of a free action of a finite group $H$ on a homotopy sphere $\Sigma(2 n+1)$. We show that for a map $f: A \rightarrow \Sigma(2 n+1)$ with $1<\operatorname{dim} A=d \leqslant 2 n+1, \pi_{k}(A)=0$ for $1<k<2 n+1$ and $H^{2 n+1}\left(\pi_{1}(A) ; \mathbb{Z}\right)=0$, we have $G_{1}^{f}(\Sigma(2 n+1) / H)=\mathcal{Z}_{H} f_{*}\left(\pi_{1}(A)\right)$, the centralizer of $f_{*}\left(\pi_{1}(A)\right)$ in $H$. In particular, we obtain that the Jiang group obeys $J(f)=\mathcal{Z}_{H} f_{*}(H)$ for any $f: \Sigma(2 n+1) / H \rightarrow \Sigma(2 n+1) / H$.

Further, we compute or estimate the Gottlieb groups $G_{m}^{f}(\Sigma(2 n+1) / H)$ and $P_{m}^{f}(\Sigma(2 n+1) / H)$ for certain $m>1$, finite groups $H$ and $f: A \rightarrow \Sigma(2 n+1) / H$.

In Section 2, Proposition 2.4 generalizes Gottlieb's result [11] and states that $G_{1}^{f}(K(\pi, 1))=\mathcal{Z}_{\pi} f_{*}\left(\pi_{1}(A)\right)$ for $f: A \rightarrow K(\pi, 1)$ and Theorem 2.10 states:

If $p: \tilde{X} \rightarrow X$ is a covering map of a space $X$ and $f: A \rightarrow \tilde{X}$ then the isomorphism $p_{*}: \pi_{m}(\tilde{X}) \rightarrow \pi_{m}(X)$ for $m>1$ restricts to isomorphisms

$$
p_{* \mid}: G_{m}^{f}(\tilde{X}) \rightarrow G_{m}^{p f}(X) \text { and } p_{* \mid}: P_{m}^{f}(\tilde{X}) \rightarrow P_{m}^{p f}(X)
$$

for $m>1$.

The main result of this section, generalizing [20, Theorem A], is Theorem 2.17 which implies that

$$
G_{1}^{f}(\Sigma(2 n+1) / H)=\mathcal{Z}_{H} f_{*}(K)
$$

for $f: \Sigma(2 d+1) / K \rightarrow \Sigma(2 n+1) / H$ with $d \leqslant n$. Then, in Corollary 2.19, for the quotient map $\gamma_{2 n+1}: \Sigma(2 n+1) \rightarrow \Sigma(2 n+1) / H$, we have:

(1) $P_{1}^{\gamma_{2 n+1}}(\Sigma(2 n+1) / H)=G_{1}^{\gamma_{2 n+1}}(\Sigma(2 n+1) / H)=H$ provided $H$ is abelian;

(2) $P_{m}^{\gamma_{2 n+1}}(\Sigma(2 n+1) / H)=\gamma_{2 n+1 *}\left(P_{m}(\Sigma(2 n+1))\right)=\gamma_{2 n+1 *}\left(G_{m}\left(\mathbb{S}^{2 n+1}\right)\right)$ for $m>1$.

Section 3 is devoted to Whitehead center groups of projective spaces and some space forms. Subsection 3.1 makes use of some results from [10] to take up the systematic study of the groups $G_{m}\left(\Sigma(2 n+1) / \mathbb{Z}_{l}\right)$ for certain $m>1$.

Finally, Subsection 3.2 applies [10] to present computations of $G_{m}\left(\mathbb{S}^{2 n+1} / H\right)$ for $m>1$ and $H<\mathbb{S}^{3}$.

\section{Generalized Gottlieb and Whitehead center groups}

Given spaces $A$ and $X$, write $X^{A}$ for the space of continuous unbased maps from $A$ into $X$ with the compact-open topology. Write ev $(g)=g\left(a_{0}\right)$ for the basepoint $a_{0} \in A$. Let $f: A \rightarrow X$ with $f\left(a_{0}\right)=x_{0} \in X$ and consider the component of $X^{A}$ containing $f$, 
$\left(X^{A}, f\right)$. Then

$$
G_{m}^{f}(X)=\operatorname{Im}\left(\mathrm{ev}_{*}: \pi_{m}\left(X^{A}, f\right) \rightarrow \pi_{m}\left(X, x_{0}\right)\right)
$$

for $m \geqslant 1$.

If $f \in P_{n}(X)$ for some $n \geqslant 1$ then $G_{m}^{f}(X)=P_{m}^{f}(X)=\pi_{m}(X)$ for any $m \geqslant 1$ because the Whitehead product $[\alpha, f]$ is the obstruction to extending the map $\mathbb{S}^{m} \mathrm{~V}$ $\mathbb{S}^{n} \stackrel{\alpha \vee f}{\longrightarrow} X$ to $\mathbb{S}^{m} \times \mathbb{S}^{n} \rightarrow X$ for $\alpha \in \pi_{m}(X)$.

Further, we have the following:

Proposition 2.1. Let $f: A \rightarrow X$. Then:

(1) any map $g: A^{\prime} \rightarrow A$ leads to the inclusion relations $G_{m}^{f}(X) \subseteq G_{m}^{f g}(X)$ and $P_{m}^{f}(X) \subseteq P_{m}^{f g}(X)$ for $m \geqslant 1$

(2) if $g: A^{\prime} \rightarrow A$ is a homotopy equivalence then $G_{m}^{f}(X)=G_{m}^{f g}(X)$ and $P_{m}^{f}(X)=$ $P_{m}^{f g}(X)$ for $m \geqslant 1$;

(3) if $f_{*}: \pi_{1}(A) \rightarrow \pi_{1}(X)$ is the induced homomorphism by a map $f: A \rightarrow X$ and $\pi_{1}(X)$ acts trivially on $\pi_{m}(X)$ for all $m>1$ then $P_{1}^{f}(X)=\mathcal{Z}_{\pi_{1}(X)} f_{*}\left(\pi_{1}(A)\right)$, the centralizer of the image $f_{*}\left(\pi_{1}(A)\right)$ in $\pi_{1}(X)$. If $f_{*}: \pi_{*}(A) \rightarrow \pi_{*}(X)$ is an epimorphism then $P_{m}^{f}(X)=P_{m}(X)$ for all $m \geqslant 1$;

(4) given a map $h: X \rightarrow Y$ the induced homomorphism $h_{*}: \pi_{m}(X) \rightarrow \pi_{m}(Y)$ restricts to homomorphisms $h_{* \mid}: G_{m}^{f}(X) \rightarrow G_{m}^{h f}(Y)$ and $h_{* \mid}: P_{m}^{f}(X) \rightarrow P_{m}^{h f}(Y)$. In particular, for $f=\mathrm{id}_{X}$, we obtain homomorphisms $h_{* \mid}: G_{m}(X) \rightarrow G_{m}^{h}(Y)$ and $h_{* \mid}: P_{m}(X) \rightarrow P_{m}^{h}(Y)$. Because $G_{m}(Y) \subseteq G_{m}^{h}(Y)$ and $P_{m}(Y) \subseteq P_{m}^{h}(Y)$, we conclude that $h_{* \mid}^{-1}\left(G_{m}(Y)\right) \subseteq G_{m}(X)$ and $h_{* \mid}^{-1}\left(P_{m}(Y)\right) \subseteq P_{m}(X) ;$

(5) if $A=\mathbb{S}^{k}$ then $G_{m}^{f}(X)=P_{m}^{f}(X)=\operatorname{Ker}[f,-]$ for $m \geqslant 1$;

(6) if $\alpha: \mathbb{S}^{l} \rightarrow \mathbb{S}^{n}$ then the induced map $\alpha^{*}: \pi_{n}(X) \rightarrow \pi_{l}(X)$ restricts to maps

$$
\alpha_{\mid}^{*}: G_{n}^{f}(X) \rightarrow G_{l}^{f}(X) \text { and } \alpha_{\mid}^{*}: P_{n}^{f}(X) \rightarrow P_{l}^{f}(X) .
$$

If a map $\alpha=E \beta$ is a suspension, then:

(i) the restricted maps $\alpha_{\mid}^{*}$ are homomorphisms;

(ii) $\left(E^{m-n} \alpha\right)^{*}: G_{m}^{\alpha}\left(\mathbb{S}^{n}\right) \rightarrow G_{m-n+l}\left(\mathbb{S}^{n}\right)$ for $m \geqslant n$.

Proof. Because (1)-(6)(i) are obvious, we show only (6)(ii). We write $E$ for the suspension functor on the category of pointed spaces and apply the following property [25, Chapter X, (8.18) Theorem] of the Whitehead product:

$$
\left[\gamma E \delta, \gamma^{\prime} E \delta^{\prime}\right]=\left[\gamma, \gamma^{\prime}\right] E\left(\delta \wedge \delta^{\prime}\right)
$$

for $\gamma \in \pi_{s}(X), \gamma^{\prime} \in \pi_{t}(X)$ and maps $\delta: \mathbb{S}^{r-1} \rightarrow \mathbb{S}^{s-1}$ and $\delta^{\prime}: \mathbb{S}^{r^{\prime}-1} \rightarrow \mathbb{S}^{t-1}$.

Now, notice that we may assume $l, m \geqslant n$ and write $\iota_{k}$ for the identity map of the sphere $\mathbb{S}^{k}$. Then, given $\nu \in G_{m}^{\alpha}\left(\mathbb{S}^{n}\right)$, we obtain:

$$
\begin{aligned}
0 & =[\nu, \alpha]=\left[\nu E \iota_{m-1}, \iota_{n} E \beta\right]=\left[\nu, \iota_{n}\right] E\left(\iota_{m-1} \wedge \beta\right) \\
& =\left[\nu, \iota_{n}\right] E^{m} \beta=\left[\nu, \iota_{n}\right] E\left(E^{m-n} \beta \wedge \iota_{n-1}\right)=\left[\nu E^{m-n+1} \beta, \iota_{n}\right]=\left[\nu E^{m-n} \alpha, \iota_{n}\right] .
\end{aligned}
$$

Consequently,

$$
\left(E^{m-n} \alpha\right)_{\mid}^{*}: G_{m}^{\alpha}\left(\mathbb{S}^{n}\right) \rightarrow G_{m-n+l}\left(\mathbb{S}^{n}\right)
$$

and the proof is complete. 
Example 2.2.

(1) Recall (see e.g., [10, Chapter 1, (1.2)]) that the order of the Whitehead product

$$
\sharp\left[\iota_{n}, \iota_{n}\right]= \begin{cases}1, & \text { for } n=1,3,7 ; \\ 2, & \text { for } n \neq 1,3,7 \text { odd; } \\ \infty, & \text { for } n \text { even. }\end{cases}
$$

This implies that

$$
G_{n}\left(\mathbb{S}^{n}\right)=P_{n}\left(\mathbb{S}^{n}\right)= \begin{cases}\pi_{n}\left(\mathbb{S}^{n}\right), & \text { for } n=1,3,7 \\ 2 \pi_{n}\left(\mathbb{S}^{n}\right), & \text { for } n \neq 1,3,7 \text { odd }\end{cases}
$$

and $G_{n}\left(\mathbb{S}^{n}\right)=0$ provided $n$ is even.

Given a map $f: \mathbb{S}^{n} \rightarrow \mathbb{S}^{n}$, write $\operatorname{deg} f$ for its degree and notice that $\alpha \in$ $G_{n}^{f}\left(\mathbb{S}^{n}\right)=P_{n}^{f}\left(\mathbb{S}^{n}\right)$ if and only if $(\operatorname{deg} f) \alpha \in G_{n}\left(\mathbb{S}^{n}\right)$. Applying the order of $\left[\iota_{n}, \iota_{n}\right]$, we deduce that

$$
G_{n}^{f}\left(\mathbb{S}^{n}\right)=P_{n}^{f}\left(\mathbb{S}^{n}\right)= \begin{cases}\pi_{n}\left(\mathbb{S}^{n}\right), & \text { for } \operatorname{deg} f \text { even; } \\ G_{n}\left(\mathbb{S}^{n}\right), & \text { for } \operatorname{deg} f \text { odd }\end{cases}
$$

if $n$ is odd. Further, $G_{n}^{f}\left(\mathbb{S}^{n}\right)=0$ provided $n$ is even.

(2) Consider a finite group $H$ with a free action $H \times \mathbb{S}^{n} \rightarrow \mathbb{S}^{n}$ for an odd $n \geqslant 1$, write $\mathbb{S}^{n} / H$ for the associated orbit space and $\gamma: \mathbb{S}^{n} \rightarrow \mathbb{S}^{n} / H$ for the quotient map.

Let $f: \mathbb{S}^{k} \rightarrow \mathbb{S}^{n} / H$ be a pointed map with $k \geqslant 1$. Notice that for $k>1$ there is a unique map $f^{\prime}: \mathbb{S}^{k} \rightarrow \mathbb{S}^{n}$ with $f=\gamma f^{\prime}$. It is well-known (see e.g., [6, Chapter VII, Proposition 10.2]) that $\pi_{1}\left(\mathbb{S}^{n} / H\right) \cong H$ acts trivially on $\pi_{m}\left(\mathbb{S}^{n} / H\right) \cong$ $\pi_{m}\left(\mathbb{S}^{n}\right)$ for $m>1$. Then, by Proposition 2.1(5), we get:

$$
G_{m}^{f}\left(\mathbb{S}^{n} / H\right)=P_{m}^{f}\left(\mathbb{S}^{n} / H\right)=\operatorname{Ker}[f,-]= \begin{cases}\gamma_{*} G_{m}^{f^{\prime}}\left(\mathbb{S}^{n}\right), & \text { if } k>1 \\ \gamma_{*} \pi_{m}\left(\mathbb{S}^{n}\right)=\pi_{m}\left(\mathbb{S}^{n} / H\right), & \text { if } k=1,\end{cases}
$$

for $m>1$ and

$$
G_{1}^{f}\left(\mathbb{S}^{n} / H\right)=P_{1}^{f}\left(\mathbb{S}^{n} / H\right)= \begin{cases}H, & \text { if } k>1 \\ \mathcal{Z}_{H}\langle f\rangle, & \text { if } k=1\end{cases}
$$

Now, we show:

Lemma 2.3. If $f: A \rightarrow X$ then $P_{1}^{f}(X) \subseteq \mathcal{Z}_{\pi_{1}(X)} f_{*}\left(\pi_{1}(A)\right)$.

Proof. Take $\alpha \in P_{1}^{f}(X)$. Then, the Whitehead product $\left[\alpha, f_{*}(\beta)\right]=0$ for all $\beta \in$ $\pi_{1}(A)$. This implies $\alpha f_{*}(\beta)=f_{*}(\beta) \alpha$ and the proof follows.

Because $G_{1}^{f}(X) \subseteq P_{1}^{f}(X)$, we see that

$$
G_{1}^{f}(X) \subseteq \mathcal{Z}_{\pi_{1}(X)} f_{*}\left(\pi_{1}(A)\right),
$$

which for $f=\operatorname{id}_{X}$ implies the result of Gottlieb [11].

Gottlieb [11, Corollary I.13] has shown that $G_{1}(K(\pi, 1))=\mathcal{Z} \pi$, the center of the group $\pi$. We generalize that result as follows: 
Proposition 2.4. If $f: A \rightarrow K(\pi, 1)$ then $G_{1}^{f}(K(\pi, 1))=\mathcal{Z}_{\pi} f_{*}\left(\pi_{1}(A)\right)$.

Proof. By the above, we have $G_{1}^{f}(K(\pi, 1)) \subseteq \mathcal{Z}_{\pi} f_{*}\left(\pi_{1}(A)\right)$.

To show the opposite inclusion, take $\alpha \in \mathcal{Z}_{\pi} f_{*}\left(\pi_{1}(A)\right)$ and consider the homomorphism $\varphi: \pi_{1}\left(A \times \mathbb{S}^{1}\right)=\pi_{1}(A) \times \mathbb{Z} \rightarrow \pi$ given by $\varphi(g, n)=\alpha^{n} f_{*}(g)$ for $(g, n) \in$ $\pi_{1}(A) \times \mathbb{Z}$, where $\mathbb{Z}$ is the group of integers. Since homotopy classes of maps into a $K(\pi, 1)$ are determined at the fundamental group level (see, e.g., [25, Chapter V, (4.3) Theorem]), we obtain the required map $A \times \mathbb{S}^{1} \rightarrow K(\pi, 1)$.

We point out that a similar result as stated above has been already obtained in $[\mathbf{1 3}$, Lemma 2] and the inclusion $\mathcal{Z}_{\pi} f_{*}(\pi) \subseteq G_{1}^{f}(K(\pi, 1))$ for any self-map $f: K(\pi, 1) \rightarrow$ $K(\pi, 1)$ shown in [7, Chapter VII, Theorem 10].

Example 2.5. (1) If $\pi$ is an abelian group then $G_{1}(K(\pi, 1))=G_{1}^{f}(K(\pi, 1))=\pi$ for any $f: A \rightarrow K(\pi, 1)$.

(2) Let $Q_{8}=\langle i, j\rangle$ be the quaternionic group. Because the center $\mathcal{Z}\left(Q_{8}\right)=\mathbb{Z}_{2}$, we see that $G_{1}\left(K\left(Q_{8}, 1\right)\right)=\mathbb{Z}_{2}$. Let $f: \mathbb{S}^{1} \rightarrow K\left(Q_{8}, 1\right)$ be the map determined by the homomorphism $\mathbb{Z} \rightarrow Q_{8}$ such that $1 \mapsto i$. Then, $G_{1}^{f}\left(K\left(Q_{8}, 1\right)\right)=\mathcal{Z}_{Q_{8}}\langle i\rangle=$ $\langle i\rangle$ and so we have the proper inclusion $G_{1}\left(K\left(Q_{8}, 1\right)\right) \varsubsetneqq G_{1}^{f}\left(K\left(Q_{8}, 1\right)\right)$.

Recall that a space $X$ is said to be aspherical if $\pi_{m}(X, x)=0$ for $m>1$ and all $x \in$ $X$. Following the ideas stated in $\left[\mathbf{1 1}\right.$, Section III, $\left.X^{X}\right]$, we can easily generalize $[\mathbf{1 1}$, Theorem III.2], [13, Lemma 2] and [15, Theorems 6.1 and 6.2] as follows:

Proposition 2.6. If $X$ is a locally finite, aspherical, path-connected space and $f: A \rightarrow X$ then:

(1) $\mathrm{ev}_{*}: \pi_{1}\left(X^{A}, f\right) \stackrel{\cong}{\rightarrow} G_{1}^{f}(X)=\mathcal{Z}_{\pi_{1}(X)} f_{*}\left(\pi_{1}(A)\right)$;

(2) $\pi_{m}\left(X^{A}, f\right)=0$ for $m>1$.

In particular, if $A$ is a 1-connected space then the space $X^{A}$ is path-connected and $\pi_{1}\left(X^{A}, f\right) \cong G_{1}^{f}(X)=\pi_{1}(X)$ for any $f: A \rightarrow X$ provided $X$ is a locally finite, aspherical and path-connected space.

Further, Gottlieb [12, Theorems 6-1 and 6-2] has shown:

Proposition 2.7. If $p: \tilde{X} \rightarrow X$ is a covering map then

$$
p_{*}^{-1}\left(G_{m}(X)\right) \subseteq G_{m}(\tilde{X}) \text { for } m \geqslant 1 .
$$

For Whitehead center groups, we have:

Proposition 2.8. If $p: \tilde{X} \rightarrow X$ is a covering map then

$$
p_{*}^{-1}\left(P_{m}(X)\right) \subseteq P_{m}(\tilde{X}) \text { for } m \geqslant 1 .
$$

If $X$ is a simple space then

$$
p_{*}^{-1}\left(P_{m}(X)\right)=P_{m}(\tilde{X}) \text { for } m \geqslant 1 .
$$

Proof. Since $p_{*}: \pi_{*}(\tilde{X}) \rightarrow \pi_{*}(X)$ is a monomorphism, the inclusion $p_{*}^{-1}\left(P_{m}(X)\right) \subseteq$ $P_{m}(\tilde{X})$ for $m \geqslant 1$ is straightforward. Let now $X$ be a simple space and take $\alpha \in$ $P_{m}(\tilde{X})$, and $\beta \in \pi_{k}(X)$. If $k=1$ then $[p \alpha, \beta]=0$ since $X$ is a simple space. If $k>1$ then there is $\gamma \in \pi_{k}(\tilde{X})$ such that $p \gamma=\beta$. Hence, $[p \alpha, \beta]=[p \alpha, p \gamma]=p_{*}[\alpha, \gamma]=0$ and the proof follows. 
To state the next result, we prove:

Lemma 2.9. If $p: \tilde{X} \rightarrow X$ is a covering map and $f: A \rightarrow \tilde{X}$ then:

(1) $G_{1}^{f}(\tilde{X})=p_{*}^{-1}\left(G_{1}^{p f}(X)\right)$;

(2) $P_{1}^{f}(\tilde{X})=p_{*}^{-1}\left(P_{1}^{p f}(X)\right)$.

Proof. Because $p_{*}: \pi_{1}(\tilde{X}) \rightarrow \pi_{1}(X)$ restricts to $p_{* \mid}: G_{1}^{f}(\tilde{X}) \rightarrow G_{1}^{p f}(X)$ and $p_{* \mid}: P_{1}^{f}(\tilde{X})$ $\rightarrow P_{1}^{p f}(X)$, we deduce that $G_{1}^{f}(\tilde{X}) \subseteq p_{*}^{-1}\left(G_{1}^{p f}(X)\right)$ and $P_{1}^{f}(\tilde{X}) \subseteq p_{*}^{-1}\left(P_{1}^{p f}(X)\right)$.

(1): We prove $p_{*}^{-1}\left(G_{1}^{p f}(X)\right) \subseteq G_{1}^{f}(\tilde{X})$. Given $\alpha \in p_{*}^{-1}\left(G_{1}^{p f}(X)\right)$, we have a map $F: A \times \mathbb{S}^{1} \rightarrow X$ such that the diagram

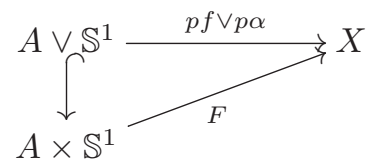

commutes up to homotopy. But, for any $\beta: \mathbb{S}^{1} \rightarrow A \times \mathbb{S}^{1}$ there is a map $\beta^{\prime}: \mathbb{S}^{1} \rightarrow$ $A \vee \mathbb{S}^{1}$ such that the composition $\mathbb{S}^{1} \stackrel{\beta^{\prime}}{\longrightarrow} A \vee \mathbb{S}^{1} \stackrel{i}{\hookrightarrow} A \times \mathbb{S}^{1}$ is homotopic to $\beta$. Then, $F \beta=p(f \vee \alpha) \beta^{\prime}$ and, consequently, $F_{*}\left(\pi_{1}\left(A \times \mathbb{S}^{1}\right)\right) \subseteq p_{*}\left(\pi_{1}(\tilde{X})\right)$. Hence, there is a lifting $\tilde{F}: A \times \mathbb{S}^{1} \rightarrow \tilde{X}$ of the map $F$. Because $p \tilde{F}_{\mid A \vee \mathbb{S}^{1}}=F_{\mid A \vee \mathbb{S}^{1}}=p(f \vee \alpha)$, we see that $\tilde{F}_{\mid A \vee \mathbb{S}^{1}}=f \vee \alpha$ and so $\alpha \in G_{1}^{f}(\tilde{X})$.

(2): We show $p_{*}^{-1}\left(P_{1}^{p f}(X)\right) \subseteq P_{1}^{f}(\tilde{X})$. Given $\alpha \in p_{*}^{-1}\left(P_{1}^{p f}(X)\right)$, we have $p \alpha \in$ $P_{1}^{p f}(X)$. Then, for any $\gamma \in \pi_{m}(A)$ with $m \geqslant 1$, we obtain $p_{*}[\alpha, f \gamma]=[p \alpha, p f \gamma]=0$. Because $p_{*}$ is a monomorphism, $[\alpha, f \gamma]=0$ and so $\alpha \in P_{1}^{f}(\tilde{X})$ and the proof follows.

Theorem 2.10. If $p: \tilde{X} \rightarrow X$ is a covering map and $f: A \rightarrow \tilde{X}$ then the monomorphism $p_{*}: \pi_{m}(\tilde{X}) \rightarrow \pi_{m}(X)$ for $m \geqslant 1$ yields:

(1) $G_{m}^{f}(\tilde{X})=p_{*}^{-1}\left(G_{m}^{p f}(X)\right)$;

(2) $P_{m}^{f}(\tilde{X})=p_{*}^{-1}\left(P_{m}^{p f}(X)\right)$.

Proof. In view of Lemma 2.9, we may assume that $m>1$. Because $p_{*}: \pi_{m}(\tilde{X}) \rightarrow$ $\pi_{m}(X)$ is an isomorphism, we see that its restrictions

$$
p_{* \mid}: G_{m}^{f}(\tilde{X}) \rightarrow G_{m}^{p f}(X) \text { and } p_{* \mid}: P_{m}^{f}(\tilde{X}) \rightarrow P_{m}^{p f}(X)
$$

are monomorphisms.

First, we prove that $p_{* \mid}: G_{m}^{f}(\tilde{X}) \rightarrow G_{m}^{p f}(X)$ is surjective for $m>1$. Given $\alpha \in$ $G_{m}^{p f}(X)$, there are $\beta \in \pi_{m}(\tilde{X})$ such that $p \beta=\alpha$ and $F: A \times \mathbb{S}^{m} \rightarrow X$ extending $p f \vee$ $\alpha: A \vee \mathbb{S}^{m} \rightarrow X$. But the 2-skeleton $\left(A \times \mathbb{S}^{m}\right)^{(2)}=A^{(2)} \vee\left(\mathbb{S}^{m}\right)^{(2)}$, so we obtain that $\pi_{1}\left(A \times \mathbb{S}^{m}\right)=\pi_{1}\left(A^{(2)} \vee\left(\mathbb{S}^{m}\right)^{(2)}\right)=\pi_{1}\left(A^{(2)}\right)=\pi_{1}(A)$. Because $F_{\mid A \vee \mathbb{S}^{m}}=p f \vee \alpha$, this implies that $F_{*}\left(\pi_{1}\left(A \times \mathbb{S}^{m}\right)\right)=(p f)_{*}\left(\pi_{1}(A)\right) \subseteq p_{*}\left(\pi_{1}(\tilde{X})\right)$. Hence, the map $F: A \times$ $\mathbb{S}^{m} \rightarrow X$ lifts to $\tilde{F}: A \times \mathbb{S}^{m} \rightarrow \tilde{X}$. Because $p \tilde{F}_{\mid A \vee \mathbb{S}^{m}}=F_{\mid A \vee \mathbb{S}^{m}}=p(f \vee \beta)$, the map $\tilde{F}$ extends $f \vee \beta: A \vee \mathbb{S}^{m} \rightarrow \tilde{X}$ and so $\beta \in G_{m}^{f}(\tilde{X})$.

Now, we show that $p_{* !}: P_{m}^{f}(\tilde{X}) \rightarrow P_{m}^{p f}(X)$ is surjective for $m>1$. Given $\alpha \in$ $P_{m}^{p f}(X)$, there is $\beta \in \pi_{m}(\tilde{X})$ such that $p \beta=\alpha$. Then, $p_{*}[\beta, f \gamma]=[p \beta, p f \gamma]=[\alpha, p f \gamma]$ $=0$ for any $\gamma \in \pi_{l}(\tilde{X})$ which implies $[\beta, f \gamma]=0$ and so $\beta \in P_{m}^{f}(\tilde{X})$, and the proof is complete. 
Then, Proposition 2.8 and Theorem 2.10 yield:

Corollary 2.11. If $p: \tilde{X} \rightarrow X$ is a covering map then $G_{m}(\tilde{X})=p_{*}^{-1}\left(G_{m}^{p}(X)\right)$ and $P_{m}(\tilde{X})=p_{*}^{-1}\left(P_{m}^{p}(X)\right)$ for $m \geqslant 1$. Further, if $X$ is a simple space then $P_{m}(\tilde{X})=$ $p_{*}^{-1}\left(P_{m}^{p}(X)\right)=p_{*}^{-1}\left(P_{m}(X)\right)$ for $m \geqslant 1$.

To extend [20, THEOREM A] and state our main result, we need some prerequisites. Let $\pi$ be an abelian group. Given $\Phi: A \times \mathbb{S}^{1} \rightarrow X$, write $f=\Phi_{\mid A}$ for its restriction to the space $A$. The induced map $\Phi^{*}: H^{n}(X ; \pi) \rightarrow H^{n}\left(A \times \mathbb{S}^{1} ; \pi\right)$ gives

$$
\Phi^{*}(x)=f^{*}(x) \otimes 1+x_{\Phi} \otimes \lambda,
$$

where $x \in H^{n}(X ; \pi)$, the element $\lambda$ is a chosen generator of $H^{1}\left(\mathbb{S}^{1} ; \mathbb{Z}\right)$ and $x_{\Phi} \in$ $H^{n-1}(A ; \pi)$.

Now, take an integer $m>0$. Recall that a map $p: E \rightarrow B$ is called a principal $K(\pi, m)$-fibration if it is a pullback of the path fibration $K(\pi, m) \rightarrow P K(\pi, m+1) \rightarrow$ $K(\pi, m+1)$ via the classifying map $k: B \rightarrow K(\pi, m+1)$. If $\iota \in H^{m+1}(K(\pi, m+$ $1) ; \pi)$ is the fundamental class of $K(\pi, m+1)$, let $k^{*}(\iota)=\mu \in H^{m+1}(B ; \pi)$ and recall that a map $\varphi: Y \rightarrow B$ has a lifting $\bar{\varphi}: Y \rightarrow E$ if and only if $\varphi^{*}(\mu)=0$.

Then, following mutatis mutandis the proof of [20, THEOREM 1], we can show a fundamental lifting result due to Gottlieb [12]:

Lemma 2.12. Let $p: E \rightarrow B$ and $p^{\prime}: E^{\prime} \rightarrow B^{\prime}$ be principal $K(\pi, m)$ - and $K\left(\pi^{\prime}, m\right)$ fibrations, respectively with a commutative diagram

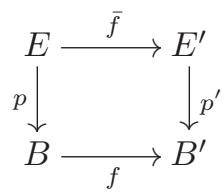

and $\Phi: B \times \mathbb{S}^{1} \rightarrow B^{\prime}$ such that $\Phi_{\mid B}=f$. If $\mu^{\prime}=k^{\prime *}\left(\iota^{\prime}\right)$, where $k^{\prime}$ classifies $p^{\prime}$ and $\iota^{\prime}$ is the fundamental class of $K\left(\pi^{\prime}, m+1\right)$ then there exists a map $\bar{\Phi}: E \times \mathbb{S}^{1} \rightarrow E^{\prime}$ such that $\bar{\Phi}_{\mid E}=\bar{f}$ and the diagram

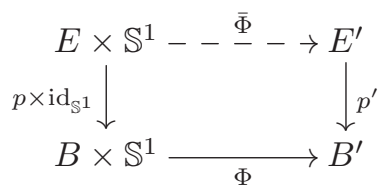

commutes if and only if $\mu_{\Phi}^{\prime}=0 \in H^{m}\left(B ; \pi^{\prime}\right)$ with $\Phi^{*}\left(\mu^{\prime}\right)=f^{*}\left(\mu^{\prime}\right) \otimes 1+\mu_{\Phi}^{\prime} \otimes \lambda$ for a chosen generator $\lambda$ of $H^{1}\left(\mathbb{S}^{1} ; \mathbb{Z}\right)$.

Remark 2.13. As in [20, REMARK, p. 68], we notice that without loss of generality we can take the diagrams to be homotopy commutative.

Next, given a space $X$, consider its universal covering $p: \tilde{X} \rightarrow X$. As usual, we can take its classifying map $\kappa: X \rightarrow K\left(\pi_{1}(X), 1\right)$ to be an inclusion. If $\pi_{1}(X)$ acts trivially on $\pi_{m}(X)$ for $m>1$ then the pair $\left(K\left(\pi_{1}(X), 1\right), X\right)$ is simple. Hence, according 
to [3, Chapter 7, Section 7.4], the Moore-Postnikov tower

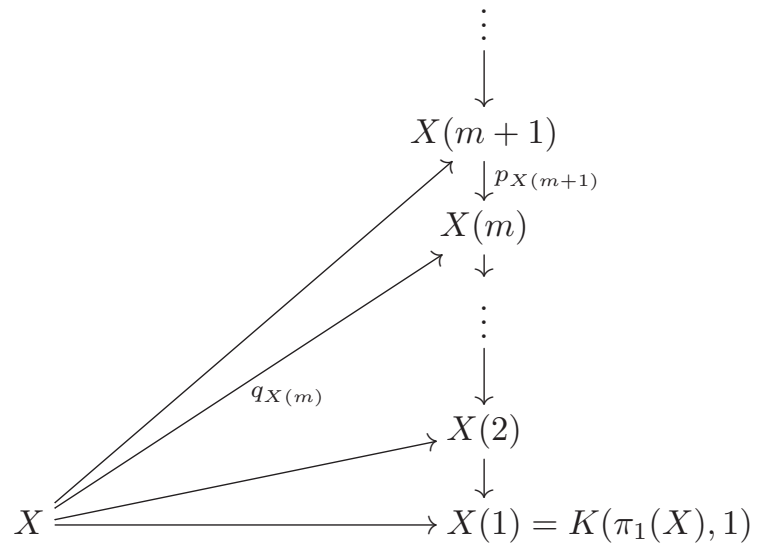

for the classifying map $\kappa: X \rightarrow K\left(\pi_{1}(X), 1\right)$ of the covering $p$ exists, where $X(m)$ is called the $m^{\text {th }}$ stage of this tower for $m \geqslant 1$. Recall that the map $q_{X(m)}: X \rightarrow X(m)$ is an $(m+1)$-equivalence for $m \geqslant 1$.

From now on, we assume that $A$ and $X$ are spaces such that $\pi_{1}(A)$ and $\pi_{1}(X)$ act trivially on $\pi_{m}(A)$ and $\pi_{m}(X)$ for $m>1$, respectively. Given a map $f: A \rightarrow X$, write $f(m): A(m) \rightarrow X(m)$ for the induced map of the $m^{\text {th }}$ stages for $m \geqslant 0$.

If $\alpha \in G_{1}^{f(m+1)}(X(m+1))$ and $\bar{\Phi}: A(m+1) \times \mathbb{S}^{1} \rightarrow X(m+1)$ is the associated map then the naturality (see, e.g., [3, Proposition 7.2.11] or [18, Theorem 2.1]) of the Moore-Postnikov tower provides a homotopy commutative diagram

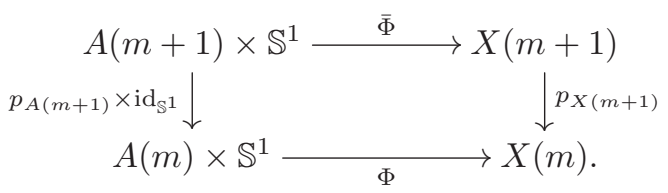

Taking $p_{A(m+1)}: A(m+1) \rightarrow A(m)$ to be an inclusion, the obstructions to the existence of a relative homotopy from $\Phi_{\mid A(m)}$ to $f(m)$ lie in $H^{k}(A(m), A(m+1)$; $\left.\pi_{k}(X(m))\right)=0$ for $k \geqslant 1$. Hence, $\Phi_{\mid A(m)}$ is homotopic to $f(m)$ and $\Phi$ is the affiliated map to $p_{X(m+1) *}(\alpha) \in G_{1}^{f(m)}(X(m))$.

Then, Lemma 2.12 leads to the following generalization of $[\mathbf{1 4}$, Lemma 7$]$ and $[\mathbf{2 0}$, TheOREM 2]:

Proposition 2.14. The map $p_{X(m+1)}: X(m+1) \rightarrow X(m)$ induces a homomorphism $p_{X(m+1) *}: G_{1}^{f(m+1)}(X(m+1)) \rightarrow G_{1}^{f(m)}(X(m))$.

Now, for $\alpha \in \mathcal{Z}_{\pi_{1}(X)} f_{*}\left(\pi_{1}(A)\right)$, consider the map $\Phi_{\alpha}: K\left(\pi_{1}(A), 1\right) \times \mathbb{S}^{1} \rightarrow K\left(\pi_{1}(X), 1\right)$ corresponding to the homomorphism

$$
\pi_{1}(A) \times \mathbb{Z} \rightarrow \pi_{1}(X)
$$

given by $(g, n) \mapsto \alpha^{n} f_{*}(g)$ for $(g, n) \in \pi_{1}(A) \times \mathbb{Z}$. 
If $H^{m}\left(A(m-1) ; \pi_{m}(X)\right)=0$ for $m>1$ then Lemma 2.12 leads to commutative diagrams

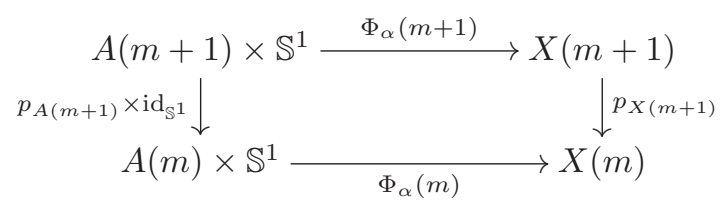

with $\Phi_{\alpha}(m)_{\mid A(m)}=f(m)$. Hence, we obtain a map

$$
\lim _{\leftarrow} \Phi_{\alpha}(m): \lim _{\leftarrow} A(m) \times \mathbb{S}^{1} \rightarrow \lim _{\leftarrow} X(m) .
$$

Let $\phi(A): A \rightarrow \lim _{\leftarrow} A(m)$ and $\phi(X): X \rightarrow \lim _{\leftarrow} X(m)$ denote the standard weak homotopy equivalences. Then, there is a unique (up to homotopy) map $\hat{\Phi}_{\alpha}: A \times \mathbb{S}^{1} \rightarrow X$ which makes the diagram

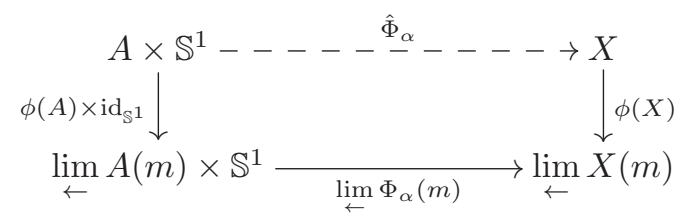

commutative. Certainly, $\hat{\Phi}_{\alpha \mid \mathbb{S}^{1}}=\alpha$. To see that $\hat{\Phi}_{\alpha \mid A}=f$, observe that if $\operatorname{dim} A=d<$ $\infty$, then there is a bijection of homotopy classes $[A, X] \cong[A, X(d)] \cong[A(d), X(d)]$ and $\hat{\Phi}_{\alpha \mid A}$ corresponds to $\Phi_{\alpha}(d)_{\mid A(d)}=f(d): A(d) \rightarrow X(d)$. Thus, this bijection implies $\hat{\Phi}_{\alpha \mid A}=f$ and we may state:

Proposition 2.15. If $f: A \rightarrow X$ with $\operatorname{dim} A=d<\infty, \alpha \in \mathcal{Z}_{\pi_{1}(X)} f_{*}\left(\pi_{1}(A)\right)$ and $H^{m}\left(A(m-1) ; \pi_{m}(X)\right)=0$ for $m>1$ then $\alpha \in G_{1}^{f}(X)$. Hence, we have $G_{1}^{f}(X)=$ $\mathcal{Z}_{\pi_{1}(X)} f_{*}\left(\pi_{1}(A)\right)$.

Since $G_{1}^{f}(X) \subseteq P_{1}^{f}(X) \subseteq \mathcal{Z}_{\pi_{1}(X)} f_{*}\left(\pi_{1}(A)\right)$, we find that

$$
P_{1}^{f}(X)=\mathcal{Z}_{\pi_{1}(X)} f_{*}\left(\pi_{1}(A)\right)
$$

under the hypothesis of Proposition 2.15.

Example 2.16. (1) If $X=K(\pi, 1)$ for some group $\pi$ then $H^{m}\left(A(m-1) ; \pi_{m}(X)\right)=$ 0 for $m>1$.

(2) If $\operatorname{dim} A=1$ then there exists a homotopy equivalence $A \simeq K(\pi, 1)$, where $\pi$ is a free group. Hence $A(m-1)=K(\pi, 1)$ and, consequently, $H^{m}(A(m-$ $\left.1) ; \pi_{m}(X)\right)=H^{m}\left(\pi ; \pi_{m}(X)\right)=0$ for $m>1$.

Now, we are in a position to state the main result of this section:

Theorem 2.17. If $f: A \rightarrow X$ with $1<\operatorname{dim} A=d<\infty$ and $H^{m}\left(A(m-1) ; \pi_{m}(X)\right)=0$ for $1<m \leqslant d$ then $G_{1}^{f}(X)=\mathcal{Z}_{\pi_{1}(X)} f_{*}\left(\pi_{1}(A)\right)$.

Proof. Since $H^{m}\left(A(m-1) ; \pi_{m}(X)\right)=0$ for $1<m \leqslant d$, in view of Proposition 2.15, we have to show that $H^{m}\left(A(m-1) ; \pi_{m}(X)\right)=0$ for $m>d$. 
Let $m>d$. Then, in view of Whitehead Theorem, it follows that the $m$-equivalence $q_{A(m-1)}: A \rightarrow A(m-1)$ induces isomorphisms $H_{k}(A ; \mathbb{Z}) \stackrel{\simeq}{\rightarrow} H_{k}(A(m-1) ; \mathbb{Z})$ for $k<$ $m$ and a surjection $H_{m}(A ; \mathbb{Z}) \rightarrow H_{m}(A(m-1) ; \mathbb{Z})$. Hence the group $H_{d}(A(d) ; \mathbb{Z})$ is free, $H_{m-1}(A(m-1) ; \mathbb{Z})=0$ for $m>d+1$ and $H_{m}(A(m-1) ; \mathbb{Z})=0$ for $m>d$. Thus, by the universal coefficient theorem, we have

$$
\begin{aligned}
H^{m}\left(A(m-1) ; \pi_{m}(X)\right) & \simeq \operatorname{Hom}\left(H_{m}(A(m-1) ; \mathbb{Z}), \pi_{m}(X)\right) \\
& \oplus \operatorname{Ext}\left(H_{m-1}(A(m-1) ; \mathbb{Z}), \pi_{m}(X)\right)=0
\end{aligned}
$$

for $m>d$ and the proof is complete.

Given a finite group $H$ acting freely and cellularly on $\mathbb{S}^{2 n+1}$ write $\mathbb{S}^{2 n+1} / H$ for the orbit space. Oprea [20, ThEOREM A] has shown that $G_{1}\left(\mathbb{S}^{2 n+1} / H\right)=\mathcal{Z} H$. Then, the relations

$$
\mathcal{Z} H=G_{1}\left(\mathbb{S}^{2 n+1} / H\right) \subseteq P_{1}\left(\mathbb{S}^{2 n+1} / H\right) \subseteq \mathcal{Z} H
$$

imply that $P_{1}\left(\mathbb{S}^{2 n+1} / H\right)=\mathcal{Z} H$, what was already observed by Gottlieb $[\mathbf{1 1}, \S 3]$ and follows from Proposition 2.1(3) as well.

Recall that a finite dimensional $C W$-complex $\Sigma(n)$ with the homotopy type of the $n$-sphere $\mathbb{S}^{n}$ is called an $n$-homotopy sphere for $n \geqslant 1$. The finite periodic groups, which are the only finite groups that can act freely and cellularly on some homotopy sphere, have been fully classified by Suzuki-Zassenhaus, see e.g., [2, Chapter IV, Theorem 6.15]. It is well-known that the only finite groups acting freely and cellularly on $\Sigma(2 n)$ are $\mathbb{Z}_{2}$ and the trivial group.

Given a free and cellular action $H \times \Sigma(2 m+1) \rightarrow \Sigma(2 m+1)$ of a finite group $H$ on a homotopy sphere $\Sigma(2 m+1)$, write $\gamma_{2 n+1}: \Sigma(2 n+1) \rightarrow \Sigma(2 n+1) / H$ for the quotient map. It is well-known [8, Chapter XVI, §4, Application 4] that $H$ must have periodic cohomology with period $2 m+2$. Then, in view of [6, Chapter VII, Proposition 10.2], the action of $H$ on $\pi_{k}(\Sigma(2 m+1) / H)$ is trivial for $k>1$. Because $H^{2 k+1}(H ; \mathbb{Z})=0$ for $k \geqslant 1$ (see e.g., [20, Lemma 6]), Theorem 2.17 yields the following generalization of $[\mathbf{2 0}$, THEOREM A]:

Corollary 2.18. If $f: A \rightarrow \Sigma(2 n+1) / H$ with $1<\operatorname{dim} A=d \leqslant 2 n+1, \pi_{k}(A)=0$ for $1<k<2 n+1$ and $H^{2 n+1}\left(\pi_{1}(A) ; \mathbb{Z}\right)=0$ then $G_{1}^{f}(\Sigma(2 n+1) / H)=\mathcal{Z}_{H} f_{*}\left(\pi_{1}(A)\right)$.

In particular, if $f: \Sigma(2 d+1) / K \rightarrow \Sigma(2 n+1) / H$ and $d \leqslant n$ then $G_{1}^{f}(\Sigma(2 n+1) / H)$ $=\mathcal{Z}_{H} f_{*}(K)$.

Proof. The first part is a direct conclusion from Theorem 2.17.

Let now $f: \Sigma(2 d+1) / K \rightarrow \Sigma(2 n+1) / H$ with $d \leqslant n$. Then $\pi_{k}(\Sigma(2 d+1) / K) \cong$ $\pi_{k}(\Sigma(2 d+1))=0$ for $1<k<2 d+1 \leqslant 2 n+1$. Next, notice that by [1] or [22] there is a homotopy sphere $\Sigma^{\prime}(2 d+1)$ admitting a free action of the group $K$ such that $\operatorname{dim} \Sigma^{\prime}(2 d+1)=2 d+1 \leqslant 2 n+1$ and the space forms $\Sigma(2 d+1) / K$ and $\Sigma^{\prime}(2 d+1) / K$ are homotopy equivalent.

Because $H^{2 n+1}(K ; \mathbb{Z})=0$ (see e.g., [20, Lemma 6$]$ ), the space $\Sigma^{\prime}(2 d+1) / K$ satisfies all required hypotheses of the first part and the proof follows. 
Suppose $f: A \rightarrow \Sigma(2 n+1) / H$ satisfies the conditions above. First, notice that

$$
G_{1}^{f}(\Sigma(2 n+1) / H)=P_{1}^{f}(\Sigma(2 n+1) / H)=\mathcal{Z}_{H} f_{*}\left(\pi_{1}(A)\right)
$$

and, if $A$ is 1-connected,

$$
G_{1}^{f}(\Sigma(2 n+1) / H)=P_{1}^{f}(\Sigma(2 n+1) / H)=H .
$$

This implies

$$
G_{1}^{f}(\Sigma(2 n+1) / H)=P_{1}^{f}(\Sigma(2 n+1) / H)=H
$$

for any map $f: \Sigma(2 d+1) \rightarrow \Sigma(2 n+1) / H$ and $1 \leqslant d \leqslant n$. Next,

$$
P_{1}^{f}(\Sigma(2 n+1) / H)=G_{1}^{f}(\Sigma(2 n+1) / H)=P_{1}(\Sigma(2 n+1) / H)=G_{1}(\Sigma(2 n+1) / H)=H
$$

provided the group $H$ is abelian.

Further, notice that given $\gamma_{2 n+1} \alpha \in P_{m}(\Sigma(2 n+1) / H)$, we have $\left[\gamma_{2 n+1} \alpha, \gamma_{2 n+1}\right]=$ $\gamma_{2 n+1}\left[\alpha, \iota_{2 n+1}\right]=0$. Since the map $\gamma_{2 n+1 *}: \pi_{m}(\Sigma(2 n+1)) \rightarrow \pi_{m}(\Sigma(2 n+1) / H)$ is a monomorphism, we see that $\alpha \in P_{m}(\Sigma(2 n+1))$ which leads to

$$
P_{m}(\Sigma(2 n+1) / H) \subseteq \gamma_{2 n+1 *} P_{m}(\Sigma(2 n+1))=\gamma_{2 n+1 *} G_{m}(\Sigma(2 n+1)) .
$$

But, $\pi_{1}(\Sigma(2 n+1) / H) \cong H$ acts trivially on $\pi_{m}(\Sigma(2 n+1) / H)$ for $m>1$. Consequently, the above yields

$$
P_{m}(\Sigma(2 n+1) / H)=\gamma_{2 n+1 *} P_{m}(\Sigma(2 n+1))=\gamma_{2 n+1 *} G_{m}(\Sigma(2 n+1))
$$

provided $H$ is abelian. Then, in view of Theorem 2.10, we have:

Corollary 2.19. Let $H \times \Sigma(2 m+1) \rightarrow \Sigma(2 m+1)$ be a free and cellular action of a finite group $H$ on a homotopy sphere $\Sigma(2 m+1)$ and let $\gamma_{2 n+1}: \Sigma(2 n+1) \rightarrow$ $\Sigma(2 n+1) / H$ be the quotient map. Then:

(1) $P_{1}^{\gamma_{2 n+1}}(\Sigma(2 n+1) / H)=G_{1}^{\gamma_{2 n+1}}(\Sigma(2 n+1) / H)=H$ provided $H$ is abelian;

(2) $G_{m}(\Sigma(2 n+1) / H) \subseteq G_{m}^{\gamma_{2 n+1}}(\Sigma(2 n+1) / H)=\gamma_{2 n+1 *} G_{m}(\Sigma(2 n+1))$ for $m>1$;

(3) we have $P_{m}(\Sigma(2 n+1) / H) \subseteq P_{m}^{\gamma_{2 n+1}}(\Sigma(2 n+1) / H)=\gamma_{2 n+1 *} P_{m}(\Sigma(2 n+1))$

$=\gamma_{2 n+1 *} G_{m}(\Sigma(2 n+1))$ for $m>1$. In particular,

$$
\begin{aligned}
P_{m}(\Sigma(2 n+1) / H)=P_{m}^{\gamma_{2 n+1}}(\Sigma(2 n+1) / H) & \\
& =\gamma_{2 n+1 *} P_{m}(\Sigma(2 n+1))=\gamma_{2 n+1 *} G_{m}(\Sigma(2 n+1))
\end{aligned}
$$

for $m>1$ provided $H$ is abelian.

Given a space $X$ and a prime number $p$, write $\pi_{m}(X ; p)$ for the $p$-primary component of $\pi_{m}(X)$. Then, the result [9, Theorem 2.3] yields:

Corollary 2.20. If $p$ is a prime not dividing the order of the finite group $H$ then

$$
\gamma_{2 n+1 *}\left(G_{m}(\Sigma(2 n+1) ; p)\right)=G_{m}(\Sigma(2 n+1) / H ; p)=\pi_{m}(\Sigma(2 n+1) / H ; p)
$$

for $m>1$.

\section{Whitehead center and Gottlieb groups of some space forms}

Throughout this section, we use the standard terminology and notations from homotopy theory, mainly from $[\mathbf{1 0}]$ and $[\mathbf{2 3}]$. We aim to compute or approximate Whitehead center groups of some space forms. 
3.1. Whitehead center groups of $\mathbb{F} P^{n}$ and Gottlieb groups of $\Sigma(2 n+1) / \mathbb{Z}_{l}$

First, let $\mathbb{F}$ denote the field of reals $\mathbb{R}$, complex numbers $\mathbb{C}$ or the skew $\mathbb{R}$-algebra of quaternions $\mathbb{H}$ and $\mathbb{F} P^{n}$ the appropriate projective $n$-space for $n \geqslant 1$.

Write $\gamma_{n}(\mathbb{F}): \mathbb{S}^{d(n+1)-1} \rightarrow \mathbb{F} P^{n}$ for the quotient map, where $d=\operatorname{dim}_{\mathbb{R}} \mathbb{F}$ and $i_{n}(\mathbb{F}): \mathbb{S}^{d}=\mathbb{F} P^{1} \hookrightarrow \mathbb{F} P^{n}$ for the canonical inclusion with $n \geqslant 1$, and recall from $[\mathbf{4}]$ that

$$
\pi_{m}\left(\mathbb{F} P^{n}\right)=\gamma_{n}(\mathbb{F})_{*} \pi_{m}\left(\mathbb{S}^{d(n+1)-1}\right) \oplus i_{n}(\mathbb{F})_{*} E \pi_{m-1}\left(\mathbb{S}^{d-1}\right) .
$$

The results below are direct consequences of Proposition 2.1(5) and the result [4, (4.1-3)] (see also [10, Lemma 2.4]).

\section{Proposition 3.1.}

(1) $G_{m}^{\gamma_{n}(\mathbb{F})}\left(\mathbb{F} P^{n}\right)=P_{m}^{\gamma_{n}(\mathbb{F})}\left(\mathbb{F} P^{n}\right)=\operatorname{Ker}\left[\gamma_{n}(\mathbb{F}),-\right]$ for $m, n \geqslant 1$.

(2) $P_{m}^{\gamma_{n}(\mathbb{R})}\left(\mathbb{R} P^{n}\right)=P_{m}\left(\mathbb{R} P^{n}\right)=\gamma_{n}(\mathbb{R})_{*} P_{m}\left(\mathbb{S}^{n}\right)$ for $m>1, n \geqslant 1$ and

$$
P_{1}^{\gamma_{n}(\mathbb{R})}\left(\mathbb{R} P^{n}\right)= \begin{cases}\pi_{1}\left(\mathbb{R} P^{n}\right), & \text { if } n \text { is odd } \\ 0, & \text { if } n \text { is even }\end{cases}
$$

(3) (i) $P_{m}^{\gamma_{n}(\mathbb{C})}\left(\mathbb{C} P^{n}\right)=P_{m}\left(\mathbb{C} P^{n}\right)=\gamma_{n}(\mathbb{C})_{*} P_{m}\left(\mathbb{S}^{2 n+1}\right)$ for $m>2$ and $n \geqslant 1$ odd, and

$$
P_{2}^{\gamma_{n}(\mathbb{C})}\left(\mathbb{C} P^{n}\right)= \begin{cases}\pi_{2}\left(\mathbb{C} P^{n}\right), & \text { if } n \text { is odd } \\ 2 \pi_{2}\left(\mathbb{C} P^{n}\right), & \text { if } n \text { is even } .\end{cases}
$$

In particular, $P_{m}^{\eta_{2}}\left(\mathbb{S}^{2}\right)=\pi_{m}\left(\mathbb{S}^{2}\right)$ for $m \geqslant 1$.

(ii) $P_{2 n+1}^{\gamma_{n}(\mathbb{C})}\left(\mathbb{C} P^{n}\right)=P_{2 n+1}\left(\mathbb{C} P^{n}\right)=\gamma_{n}(\mathbb{C})_{*} P_{2 n+1}\left(\mathbb{S}^{2 n+1}\right)$ and $2 P_{m}^{\gamma_{n}(\mathbb{C})}\left(\mathbb{C} P^{n}\right) \subseteq$ $P_{m}\left(\mathbb{C} P^{n}\right)=\gamma_{n}(\mathbb{C})_{*} P_{m}\left(\mathbb{S}^{2 n+1}\right)$ for $m>2 n+1$ and $n$ even.

(4) (i) $P_{m}^{\gamma_{n}(\mathbb{H})}\left(\mathbb{H} P^{n} ; p\right)=\gamma_{n}(\mathbb{H})_{*} \pi_{m}\left(\mathbb{S}^{4 n+3} ; p\right) \oplus i_{n}(\mathbb{H})_{*} E L_{m-1}^{\prime \prime}\left(\mathbb{S}^{3} ; p\right)$, if $p$ is an odd prime.

(ii) $P_{m}^{\gamma_{n}(\mathbb{H})}\left(\mathbb{H} P^{n} ; 2\right)=\gamma_{n}(\mathbb{H})_{*} \pi_{m}\left(\mathbb{S}^{4 n+3} ; 2\right) \oplus i_{n}(\mathbb{H})_{*} E L^{\prime \prime}\left(\mathbb{S}^{3} ; 2\right)$, provided

$$
\left[\iota_{4 n+3}, \pi_{m}^{4 n+3}\right] \cap(n+1) \nu_{4 n+3} E^{4 n+3} \pi_{m-1}^{3}=0,
$$

where $L_{m-1}^{\prime \prime}\left(\mathbb{S}^{3}\right)=\left\{\beta \in \pi_{m-1}\left(\mathbb{S}^{3}\right) ;\left[i_{n}(\mathbb{H}) E \beta, \gamma_{n}(\mathbb{H})\right]=0\right\}$. In particular,

$$
P_{m}^{\nu_{4}}\left(\mathbb{S}^{4}\right)=\left\{\begin{array}{l}
\nu_{4 *} \pi_{m}\left(\mathbb{S}^{7} ; p\right) \oplus E L_{m-1}^{\prime \prime}\left(\mathbb{S}^{3} ; p\right), \quad \text { if } p \text { is an odd prime; } \\
\nu_{4 *} \pi_{m}\left(\mathbb{S}^{7} ; 2\right) \oplus E L^{\prime \prime}\left(\mathbb{S}^{3} ; 2\right) .
\end{array}\right.
$$

(5) $P_{m}^{\sigma_{8}}\left(\mathbb{S}^{8}\right)=\left\{\begin{array}{l}\sigma_{8 *} \pi_{m}\left(\mathbb{S}^{15} ; p\right) \oplus E L_{m-1}^{\prime \prime}\left(\mathbb{S}^{7} ; p\right), \quad \text { if } p \text { is an odd prime; } \\ \sigma_{8 *} \pi_{m}\left(\mathbb{S}^{15} ; 2\right) \oplus E L^{\prime \prime}\left(\mathbb{S}^{7} ; 2\right) .\end{array}\right.$

(6) $G_{m}^{i_{n}(\mathbb{F})}\left(\mathbb{F} P^{n}\right)=P_{m}^{i_{n}(\mathbb{F})}\left(\mathbb{F} P^{n}\right)=\operatorname{Ker}\left[i_{n}(\mathbb{F}),-\right]$ for $m, n \geqslant 1$.

(7) $P_{m}^{i_{n}(\mathbb{R})}\left(\mathbb{R} P^{n}\right)=\pi_{m}\left(\mathbb{R} P^{n}\right)$ for $m, n \geqslant 1$ provided $n$ is odd, and $P_{m}^{i_{n}(\mathbb{R})}\left(\mathbb{R} P^{n}\right)=0$ for $1 \leqslant m \leqslant n$ provided $n$ is even.

(8) (i) $P_{2}^{i_{n}(\mathbb{C})}\left(\mathbb{C} P^{1}\right)=0$ and $P_{m}^{i_{n}(\mathbb{C})}\left(\mathbb{C} P^{n}\right)=\pi_{m}\left(\mathbb{C} P^{n}\right)$ for $m>2$ provided $n \geqslant 1$ is odd.

(ii) $P_{m}^{i_{n}(\mathbb{C})}\left(\mathbb{C} P^{n}\right)=\pi_{m}\left(\mathbb{C} P^{n}\right)$ for $1 \leqslant m<2 n+1, P_{2 n+1}^{i_{n}(\mathbb{C})}\left(\mathbb{C} P^{n}\right)=2 \pi_{2 n+1}\left(\mathbb{C} P^{n}\right)$ and $P_{m}^{i_{n}(\mathbb{C})}\left(\mathbb{C} P^{n}\right) \supseteq 2 \pi_{m}\left(\mathbb{C} P^{n}\right)$ for $m>2 n+1$ provided $n$ is even. 
(9) $P_{4}^{i_{n}(\mathbb{H})}\left(\mathbb{H} P^{1}\right)=0$ and $P_{m}^{i_{n}(\mathbb{H})}\left(\mathbb{H} P^{n}\right)=\pi_{m}\left(\mathbb{H} P^{n}\right)$ for $4<m<4 n+3$,

$$
P_{4 n+3}^{i_{n}(\mathbb{H})}\left(\mathbb{H} P^{n}\right)=\frac{24}{(n+1,24)} \gamma_{n}(\mathbb{H})_{*} \pi_{4 n+3}\left(\mathbb{S}^{4 n+3}\right) \oplus i_{n}(\mathbb{H})_{*} E \pi_{4 n+2}\left(\mathbb{S}^{3}\right)
$$

and $P_{m}^{i_{n}(\mathbb{H})}\left(\mathbb{H} P^{n}\right) \supseteq \frac{24}{(n+1,24)} \gamma_{n}(\mathbb{H})_{*} \pi_{m}\left(\mathbb{S}^{4 n+3}\right) \oplus i_{n}(\mathbb{H})_{*} E \pi_{m-1}\left(\mathbb{S}^{3}\right)$ for $m>4 n+3$ and $n \geqslant 1$.

Next, recall the well-known fact proved e.g., in [24] and needed in the sequel:

Theorem 3.2. If a compact Lie group $G$ acts on a compact smooth manifold $M$, then the manifold $M$ admits an equivariant triangulation. In particular, it has the structure of a $G$-CW-complex.

Consequently, any action of a finite group on the $n$-sphere $\mathbb{S}^{n}$ is cellular.

Next, given a free and cellular action $\mathbb{Z}_{2} \times \Sigma(n) \rightarrow \Sigma(n)$, by [16, Lemma 2.5], there is a homotopy equivalence $\Sigma(n) / \mathbb{Z}_{2} \simeq \mathbb{R} P^{n}$. Then, $G_{m}\left(\Sigma(n) / \mathbb{Z}_{2}\right) \cong G_{m}\left(\mathbb{R} P^{n}\right)$ and $G_{m}(\Sigma(n)) \cong G_{m}\left(\mathbb{S}^{n}\right)$ for $m \geqslant 1$. Hence, [21] implies:

$$
\begin{aligned}
G_{2 n+1}\left(\Sigma(2 n+1) / \mathbb{Z}_{2}\right) & =\gamma_{2 n+1 *} G_{2 n+1}(\Sigma(2 n+1)) \\
& = \begin{cases}\pi_{2 n+1}\left(\Sigma(2 n+1) / \mathbb{Z}_{2}\right), & \text { for } n=0,1,3 ; \\
2 \pi_{2 n+1}\left(\Sigma(2 n+1) / \mathbb{Z}_{2}\right), & \text { for odd } n \text { and } n \neq 0,1,3 .\end{cases}
\end{aligned}
$$

In virtue of the inclusion $G_{m}\left(\Sigma(2 n+1) / \mathbb{Z}_{2}\right) \subseteq \gamma_{2 n+1_{*}} G_{m}(\Sigma(2 n+1))$ for $m>1$, the group $\gamma_{2 n+1_{*}} G_{m}(\Sigma(2 n+1))$ is an upper bound of $G_{m}\left(\Sigma(2 n+1) / \mathbb{Z}_{2}\right)$.

The results in the sequel mainly follows from $[\mathbf{1 0}]$. More precisely, we make use of [10, Theorems 2.41 and 2.45, Propositions 2.42 and 2.46, and Corollary 2.47].

Theorem 3.3. If $m \leqslant 7$ then we have the equality

$$
G_{m+2 n+1}\left(\Sigma(2 n+1) / \mathbb{Z}_{2}\right)=\gamma_{2 n+1 *} G_{m+2 n+1}(\Sigma(2 n+1))
$$

except for the following pairs: $(m, 2 n+1)=\left(3,2^{l}-3\right)$ with $l \geqslant 4,\left(6,2^{l}-5\right)$ with $l \geqslant 5$ and $(7,11)$. Furthermore:

(1) $G_{2^{l}}\left(\Sigma\left(2^{l}-3\right) / \mathbb{Z}_{2}\right) \supseteq 2 \pi_{2^{l}}\left(\Sigma\left(2^{l}-3\right) / \mathbb{Z}_{2}\right)$ for $l \geqslant 4$;

(2) $G_{18}\left(\Sigma(11) / \mathbb{Z}_{2}\right) \supseteq 2 \pi_{18}\left(\Sigma(11) / \mathbb{Z}_{2}\right)$.

Remark 3.4. According to J. Mukai's conjecture,

$$
G_{18}\left(\Sigma(11) / \mathbb{Z}_{2}\right)=2 \pi_{18}\left(\Sigma(11) / \mathbb{Z}_{2}\right)=2 \gamma_{18 *} \pi_{18}(\Sigma(11))=2 \gamma_{18 *} G_{18}(\Sigma(11)) .
$$

This would imply that there is a proper inclusion $G_{18}\left(\Sigma(11) / \mathbb{Z}_{2}\right) \varsubsetneqq \gamma_{18 *} G_{18}(\Sigma(11))$.

Since $\Sigma(2 n+1) / \mathbb{Z}_{2} \simeq \mathbb{R} P^{2 n+1},[\mathbf{1 0}$, Proposition 2.42$]$ yields:

Proposition 3.5. If one of the following conditions is satisfied:

(1) $n \equiv 1(\bmod 2)$ and $m=8,9$;

(2) $n \equiv 1(\bmod 2)$ with $n \geqslant 3$ and $m=10$;

(3) $n \equiv 0(\bmod 2)$;

(4) $n \equiv 3(\bmod 4)$ with $n \geqslant 6$ and $m=11$;

(5) $n \geqslant 7$ and $m=13$ 
then $G_{m+2 n+1}\left(\Sigma(2 n+1) / \mathbb{Z}_{2}\right)=\pi_{m+2 n+1}\left(\Sigma(2 n+1) / \mathbb{Z}_{2}\right)$.

Now, we aim to study $G_{m}\left(\mathbb{S}^{2 n+1} / \mathbb{Z}_{l}\right)$ for $l>2$. First notice that the canonical fibration

$$
\mathbb{S}^{1} \rightarrow \mathbb{S}^{2 n+1} / \mathbb{Z}_{l} \rightarrow \mathbb{C} P^{n}
$$

yields an isomorphism $\pi_{m}\left(\mathbb{S}^{2 n+1} / \mathbb{Z}_{l}\right) \cong \pi_{m}\left(\mathbb{C} P^{n}\right)$ for $m \geqslant 3$.

In the sequel we need the following. Let $H$ be a closed subgroup of a Lie group $K$ and write $K / H$ for the associated orbit space. Then, $K / H \rightarrow B H \rightarrow B K$ is a fibre sequence and, by means of [12, Theorem 2-6], we obtain:

Lemma 3.6. The quotient map $p: K \rightarrow K / H$ leads to $p_{*}\left(\pi_{m}(K)\right) \subseteq G_{m}(K / H)$ for $m \geqslant 1$.

If $H$ is a finite subgroup of $K$ then Proposition 2.7 and Lemma 3.6 imply $p_{*}\left(\pi_{m}(K)\right)=G_{m}(K / H)$ for $m>1$. In particular, if $H$ is a finite subgroup of $\mathbb{S}^{3}$ then $p_{*}\left(\pi_{m}\left(\mathbb{S}^{3}\right)\right)=G_{m}\left(\mathbb{S}^{3} / H\right)$ for $m>1$.

Let now $U(n)$ be the $n^{\text {th }}$ unitary group and consider the canonical inclusion map $U(n) \times \mathbb{Z}_{l} \hookrightarrow U(n) \times U(1) \hookrightarrow U(n+1)$. Then, we may identify $\mathbb{S}^{2 n+1} \simeq U(n+1) / U(n)$ and $\mathbb{S}^{2 n+1} / \mathbb{Z}_{l} \simeq U(n+1) / U(n) \times \mathbb{Z}_{l}$. Set $p_{n}: U(n+1) \rightarrow \mathbb{S}^{2 n+1}$ and $p_{n}^{\prime}: U(n+1) \rightarrow$ $\mathbb{S}^{2 n+1} / \mathbb{Z}_{l}$ for the appropriate quotient maps being fibrations $U(n+1) \stackrel{U(n)}{\longrightarrow} \mathbb{S}^{2 n+1}$ and $U(n+1) \stackrel{U(n) \times \mathbb{Z}_{l}}{\longrightarrow} \mathbb{S}^{2 n+1} / \mathbb{Z}_{l}$, respectively. Next, consider the map of exact sequences induced by those fibrations:

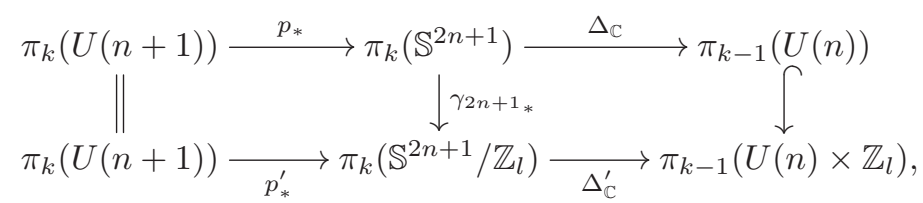

where $\Delta_{\mathbb{C}}$ and $\Delta_{\mathbb{C}}^{\prime}$ are the appropriate connecting maps. Then, by Lemma 3.6, we have:

$$
\operatorname{Ker} \Delta_{\mathbb{C}}^{\prime}=p_{*}^{\prime} \pi_{k}(U(n+1)) \subseteq G_{k}\left(\mathbb{S}^{2 n+1} / \mathbb{Z}_{l}\right) .
$$

Recall that $\pi_{m}(X ; p)$ denotes the $p$-primary component of $\pi_{m}(X)$ for a prime $p$. Let $J: \pi_{k}(S O(n)) \rightarrow \pi_{k+n}\left(\mathbb{S}^{n}\right)$ be the $J$-homomorphism and $r: U(n) \hookrightarrow S O(2 n)$ be the canonical inclusion. Taking $J_{\mathbb{C}}=J \circ r_{*}$, the result $[\mathbf{1 0}$, Lemma 2.33] implies:

\section{Proposition 3.7 .}

(1) $\operatorname{Ker}\left(\Delta_{\mathbb{C}}: \pi_{m}\left(\mathbb{S}^{2 n+1}\right) \rightarrow \pi_{m-1}(U(n))\right) \subseteq \gamma_{2 n+1 *}^{-1} G_{m}\left(\mathbb{S}^{2 n+1} / \mathbb{Z}_{l}\right)$. In particular, we have $\gamma_{2 n+1 *} \pi_{m}\left(\mathbb{S}^{2 n+1} ; p\right) \subseteq G_{m}\left(\mathbb{S}^{2 n+1} / \mathbb{Z}_{l}\right)$ provided $\pi_{m-1}(U(n) ; p)=0$ for a prime $p$.

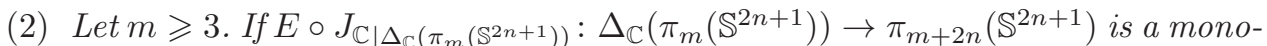
morphism then $\gamma_{2 n+1 *} G_{m}\left(\mathbb{S}^{2 n+1}\right) \subseteq G_{m}\left(\mathbb{S}^{2 n+1} / \mathbb{Z}_{l}\right)$, where $E$ is the suspension homomorphism. In particular, under this assumption, Corollary 2.19(2) yields that $G_{m}\left(\mathbb{S}^{2 n+1} / \mathbb{Z}_{l}\right)=\gamma_{2 n+1 *} G_{m}\left(\mathbb{S}^{2 n+1}\right)$.

Now, we make use of Proposition 3.7 to obtain, as [10, Theorem 2.45], the following: 


\section{Proposition 3.8.}

(1) Let $m=1,2$. Then,

$$
G_{m+2 n+1}\left(\mathbb{S}^{2 n+1} / \mathbb{Z}_{l}\right)= \begin{cases}0, & \text { if } n \text { is even }, \\ \pi_{m+2 n+1}\left(\mathbb{S}^{2 n+1} / \mathbb{Z}_{l}\right) \cong \mathbb{Z}_{2}, & \text { if } n \text { is odd. }\end{cases}
$$

(2) $G_{2 n+4}\left(\mathbb{S}^{2 n+1} / \mathbb{Z}_{l}\right) \supseteq$

$$
\begin{cases}(24, n) \pi_{2 n+4}\left(\mathbb{S}^{2 n+1} / \mathbb{Z}_{l}\right) \cong \mathbb{Z}_{\frac{24}{(24, n)}}, & \text { if } n \text { is even }, \\ \frac{(24, n+3)}{2} \pi_{2 n+4}\left(\mathbb{S}^{2 n+1} / \mathbb{Z}_{l}\right) \cong \mathbb{Z}_{\frac{48}{(24, n+3)}}, & \text { if } n \text { is odd } .\end{cases}
$$

In particular, $G_{2 n+4}\left(\mathbb{S}^{2 n+1} / \mathbb{Z}_{l}\right)=2 \pi_{2 n+4}\left(\mathbb{S}^{2 n+1} / \mathbb{Z}_{l}\right)$ for $n \equiv 2,10(\bmod 12)$ with $n \geqslant 10$ except for $n=2^{i-1}-2$ or $n \equiv 1,17(\bmod 24)$ with $n \geqslant 17$, and $G_{2 n+4}\left(\mathbb{S}^{2 n+1} / \mathbb{Z}_{l}\right)=\pi_{2 n+4}\left(\mathbb{S}^{2 n+1} / \mathbb{Z}_{l}\right)$ for $n \equiv 7,11(\bmod 12)$.

(3) $G_{2 n+6}\left(\mathbb{S}^{2 n+1} / \mathbb{Z}_{l}\right)=\pi_{2 n+6}\left(\mathbb{S}^{2 n+1} / \mathbb{Z}_{l}\right) \cong \begin{cases}0, & \text { for } n \geqslant 3, \\ \mathbb{Z}_{2}, & \text { for } n=2 .\end{cases}$

(4) $G_{2 n+7}\left(\mathbb{S}^{2 n+1} / \mathbb{Z}_{l}\right)=\pi_{2 n+7}\left(\mathbb{S}^{2 n+1} / \mathbb{Z}_{l}\right)$ for $n \equiv 2,3(\bmod 4)$.

Further, making use of Proposition 3.7, as in [10, Proposition 2.46], we can show:

Proposition 3.9. $G_{m}\left(\mathbb{S}^{7} / \mathbb{Z}_{l} ; 2\right)=\pi_{m}\left(\mathbb{S}^{7} / \mathbb{Z}_{l} ; 2\right)$ for $8 \leqslant m \leqslant 24$ unless $m=15,21$. Further, $\gamma_{7 *}\left\{\sigma^{\prime} \eta_{14}, \bar{\nu}_{7}+\varepsilon_{7}\right\} \subseteq G_{15}\left(\mathbb{S}^{7} / \mathbb{Z}_{l} ; 2\right)$ and $\gamma_{7 *}\left\{\sigma^{\prime} \sigma_{14}\right\} \subseteq G_{21}\left(\mathbb{S}^{7} / \mathbb{Z}_{l} ; 2\right)$.

Then, as in [10, Corollary 2.47], we deduce:

\section{Corollary 3.10.}

(1) $G_{m}\left(\mathbb{S}^{5} / \mathbb{Z}_{l}\right)=\pi_{m}\left(\mathbb{S}^{5} / \mathbb{Z}_{l}\right)$ for $10 \leqslant m \leqslant 12$;

(2) $G_{m}\left(\mathbb{S}^{5} / \mathbb{Z}_{l} ; p\right)=\pi_{m}\left(\mathbb{S}^{5} / \mathbb{Z}_{l} ; p\right)$ for an odd prime $p ;$

(3) $G_{m}\left(\mathbb{S}^{4 n+3} / \mathbb{Z}_{l}\right) \supseteq\left(\gamma_{4 n+3} \eta_{4 n+3}^{m}\right)_{*} \pi_{m}^{4 n+k+3}$ for $m=1,2$;

(4) (i) $G_{m}\left(\mathbb{S}^{4 n+1} / \mathbb{Z}_{l}\right) \supseteq 2(12, n)\left(\gamma_{2 n} \nu_{4 n+1}^{+}\right)_{*} \pi_{k}\left(\mathbb{S}^{4 n+4}\right)$,

(ii) $G_{m}\left(\mathbb{S}^{4 n+3} / \mathbb{Z}_{l}\right) \supseteq(12, n+2)\left(\gamma_{2 n+1} \nu_{4 n+3}^{+}\right)_{*} \pi_{m}\left(\mathbb{S}^{4 n+6}\right)$;

(5) $G_{m}\left(\mathbb{S}^{8 n+5} / \mathbb{Z}_{l}\right) \supseteq\left(\gamma_{4 n+2} \nu_{8 n+5}^{2}\right)_{*} \pi_{m}^{8 n+11}$;

(6) $G_{8 n+11}\left(\mathbb{S}^{8 n+3} / \mathbb{Z}_{l}\right)=\pi_{8 n+11}\left(\mathbb{S}^{8 n+3} / \mathbb{Z}_{l}\right)$ for $n \geqslant 2$;

(7) $G_{8 n+k}\left(\mathbb{S}^{8 n+7} / \mathbb{Z}_{l}\right)=\pi_{8 n+k}\left(\mathbb{S}^{8 n+7} / \mathbb{Z}_{l}\right)$ for $k=28,29$ with $n \geqslant 2$.

\subsection{Gottlieb groups of $\mathbb{S}^{2 n+1} / H$}

Let $H \subseteq S O(2 n+2)$ be a finite subgroup of the special orthogonal group $S O(2 n+2)$ acting freely on the $(2 n+1)$-sphere $\mathbb{S}^{2 n+1}$. Certainly, a spherical $(2 n+1)$-manifold $\mathbb{S}^{2 n+1} / H$ has a finite fundamental group isomorphic to $H$ itself. For $n=1$, Thurston's elliptization conjecture (proved by Perelman) states that, conversely, all 3-manifolds with finite fundamental group are spherical manifolds.

Then, in view of Proposition 2.7, Corollary 2.19 and Lemma 3.6, we may state:

Remark 3.11. If $H \subseteq S O(2 n+2)$ is a finite subgroup acting freely on $\mathbb{S}^{2 n+1}$ then for $m \geqslant 1$ we have:

(1) $G_{m}^{\gamma_{3}}\left(\mathbb{S}^{3} / H\right)=P_{m}^{\gamma_{3}}\left(\mathbb{S}^{3} / H\right)=G_{m}\left(\mathbb{S}^{3} / H\right)=P_{m}\left(\mathbb{S}^{3} / H\right)=\gamma_{3 *} \pi_{m}\left(\mathbb{S}^{3}\right)$ for $n=1$;

(2) $G_{m}^{\gamma_{2 n+1}}\left(\mathbb{S}^{2 n+1} / H\right)=P_{m}^{\gamma_{2 n+1}}\left(\mathbb{S}^{2 n+1} / H\right)=\gamma_{2 n+1 *} G_{m}\left(\mathbb{S}^{2 n+1}\right)$ for $n>1$. 
Next, write $V_{n, k}$ for the Stiefel manifold given by orthonormal $k$-frames in an $n$ dimensional Euclidean space and recall that $V_{n, k} \simeq O(n) / O(n-k) \simeq S O(n) / S O(n-k)$. Then, a free action of a finite subgroup $H \subseteq S O(2 n+2) \subseteq S O(2 n+3)$ on $\mathbb{S}^{2 n+1} \simeq$ $S O(2 n+2) / S O(2 n+1)$ extends to a free action on $V_{2 n+3,2} \simeq S O(2 n+3) / S O(2 n+1)$. By means of the fibration

$$
\mathbb{S}^{2 n+1} / H \rightarrow V_{2 n+3,2} / H \rightarrow \mathbb{S}^{2 n+2}
$$

for $n \geqslant 1$, it was shown in $[\mathbf{9}]$ that

$$
G_{2 n+1}\left(\mathbb{S}^{2 n+1} / H\right)= \begin{cases}\mathbb{Z}, & \text { for } n=0,1,3 ; \\ 2 \mathbb{Z}, & \text { for any other } n .\end{cases}
$$

Now, let $S p(n)$ be the $n^{\text {th }}$ symplectic group and $H<S p(1)$ be a finite subgroup. Write $i_{n}^{\prime}: S p(n) \times H \hookrightarrow S p(n) \times S p(1) \hookrightarrow S p(n+1)$ for the canonical inclusion map. Then, we may identify $\mathbb{S}^{4 n+3} \simeq S p(n+1) / S p(n)$ and $\mathbb{S}^{4 n+3} / H \simeq S p(n+1) / S p(n) \times$ $K$. Set $p_{n}: S p(n+1) \rightarrow \mathbb{S}^{4 n+3}$ and $p_{n}^{\prime}: S p(n+1) \rightarrow \mathbb{S}^{4 n+3} / H$ for the quotient maps being fibrations $S p(n+1) \stackrel{S p(n)}{\longrightarrow} \mathbb{S}^{4 n+3}$ and $S p(n+1) \stackrel{S p(n) \times H}{\longrightarrow} \mathbb{S}^{4 n+3} / H$. We consider the map of exact sequences induced by those fibrations:

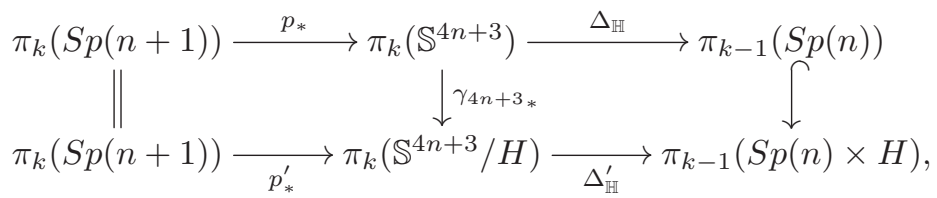

where $\Delta_{\mathbb{H}}$ and $\Delta_{\mathbb{H}}^{\prime}$ are the appropriate connecting maps. Then, by Lemma 3.6, we have:

$$
\operatorname{Ker} \Delta_{\mathbb{H}}^{\prime}=p_{*}^{\prime} \pi_{k}(S p(n+1)) \subseteq G_{k}\left(\mathbb{S}^{4 n+3} / H\right) .
$$

Write $c: S p(n) \hookrightarrow S U(2 n)$ for the canonical inclusion. Then, taking $J_{\mathbb{H}}=J \circ r_{*} \circ c_{*}$, by the result [10, Lemma 2.33], we obtain a key fact determining $G_{k}\left(\mathbb{S}^{4 n+3} / H\right)$ :

\section{Proposition 3.12.}

(1) $\operatorname{Ker}\left(\Delta_{\mathbb{H}}: \pi_{k}\left(\mathbb{S}^{4 n+3}\right) \rightarrow \pi_{k-1}(S p(n))\right) \subseteq \gamma_{4 n+3 *}^{-1} G_{k}\left(\mathbb{S}^{4 n+3} / H\right)$. In particular, we have $\gamma_{4 n+3 *} \pi_{k}\left(\mathbb{S}^{4 n+3} ; p\right) \subseteq G_{k}\left(\mathbb{S}^{4 n+3} / H\right)$ provided $\pi_{k-1}(S p(n) ; p)=0$ for a prime $p$.

(2) Let $k \geqslant 5$. If $E^{3} \circ J_{\mathbb{H} \mid \Delta_{\mathbb{H}}\left(\pi_{k}\left(\mathbb{S}^{4 n+3}\right)\right)}: \Delta_{\mathbb{H}}\left(\pi_{k}\left(\mathbb{S}^{4 n+3}\right)\right) \rightarrow \pi_{k+4 n+2}\left(\mathbb{S}^{4 n+3}\right)$ is a monomorphism then $\gamma_{4 n+3 *} G_{k}\left(\mathbb{S}^{4 n+3}\right) \subseteq G_{k}\left(\mathbb{S}^{4 n+3} / H\right)$, where $E$ is the suspension homomorphism. In particular, under this assumption, Corollary 2.19(2) yields $G_{k}\left(\mathbb{S}^{4 n+3} / H\right)=\gamma_{4 n+3 *} G_{k}\left(\mathbb{S}^{4 n+3}\right)$.

Now, we make use of Proposition 3.12 to obtain, as [10, Theorem 2.49], the following:

Theorem 3.13. $G_{k}\left(\mathbb{S}^{4 n+3} / H\right) \supseteq(24, n+2)\left(\gamma_{4 n+3} \nu_{4 n+3}^{+}\right)_{*} \pi_{k}\left(\mathbb{S}^{4 n+6}\right)$. In particular, we have $G_{4 n+6}\left(\mathbb{S}^{4 n+3} / H\right) \supseteq(24, n+2) \gamma_{4 n+3 *} \pi_{4 n+6}\left(\mathbb{S}^{4 n+3}\right) \cong \mathbb{Z}_{\frac{24}{(24, n+2)}}$ for $n \geqslant 2$.

Observe that Theorem 3.13 yields:

\section{Corollary 3.14.}

(1) $G_{8 n+9}\left(\mathbb{S}^{8 n+3} / H\right)=0$ and $G_{8 n+10}\left(\mathbb{S}^{84+7} / H\right)=\gamma_{8 n+7 *} \pi_{8 n+10}\left(\mathbb{S}^{8 n+7}\right)$ for $n \not \equiv 0$ $(\bmod 3)$. 
(2) $G_{4 n+14}\left(\mathbb{S}^{4 n+3} / H\right)=\gamma_{4 n+3 *} \pi_{4 n+14}\left(\mathbb{S}^{4 n+3}\right)$ for $n \equiv 5,9(\bmod 12)$ with $n \geqslant 5$ and $n \equiv 15,23(\bmod 24)$.

Next, applying Proposition 3.12, as [10, Corollary 2.53], we obtain:

Corollary 3.15. $G_{16 n+k}\left(\mathbb{S}^{16 n-1} / H\right)=\gamma_{16 n-1 *} \pi_{16 n+k}\left(\mathbb{S}^{16 n-1}\right)$ for $k=20, n \geqslant 1$ and $k=21, n \geqslant 2$.

Finally, as [10, Proposition 2.54], Proposition 3.12 yields:

\section{Proposition 3.16.}

(1) $G_{21}\left(\mathbb{S}^{11} / H\right) \supseteq 2 \gamma_{11 *} \pi_{21}\left(\mathbb{S}^{11}\right)$;

(2) $G_{22}\left(\mathbb{S}^{11} / H\right) \supseteq 8 \gamma_{11 *} \pi_{22}\left(\mathbb{S}^{11}\right) \cong \mathbb{Z}_{63} ;$

(3) $G_{22}\left(\mathbb{S}^{15} / H\right) \supseteq 4 \gamma_{15 *} \pi_{22}\left(\mathbb{S}^{15}\right) \cong \mathbb{Z}_{60}$.

\section{Acknowledgments}

This work was initiated and completed during the first author's visits to Instituto de Geociências e Ciências Exatas, UNESP-Univ Estadual Paulista, Rio Claro-SP, Brazil. He would like to thank the Instituto de Geociências e Ciências Exatas for its hospitality. Special thanks are due to the CAPES-Ciência sem Fronteiras, Processo: 88881.068125/2014-01 for supporting these visits.

The authors would like to thank D.L. Gonçalves for posing a possible generalization of Oprea's result [20]. Finally, they would like to express their gratitude to the anonymous referee for his/her invaluable suggestions which helped to improve the exposition of the paper.

\section{References}

[1] A. Adem and J.F. Davis, Topics in transformation groups, Handbook of geometric topology, 1-54, North-Holland, Amsterdam (2002).

[2] A. Adem and R.J. Milgram, Cohomology of finite groups, Springer, New York (1994).

[3] M. Arkowitz, Introduction to homotopy theory, Universitext, Springer, New York (2011).

[4] M.G. Barratt, I.M. James and N. Stein, Whitehead products and projective spaces, J. Math. Mech. 9 (1960), 813-819.

[5] S.A. Broughton, The Gottlieb group of finite linear quotients of odd-dimensional spheres, Proc. Amer. Math. Soc. 111, no. 4 (1991), 1195-1197.

[6] K.S. Brown, Cohomology of groups, Springer, New York (1982).

[7] R.F. Brown, The Lefschetz fixed point theorem, Scott, Foresman and Co., Glenview, Ill., London (1971).

[8] H. Cartan and S. Eilenberg, Homological algebra, Princeton (1956).

[9] M. Golasiński and D.L. Gonçalves, Postnikov towers and Gottlieb groups of orbit spaces, Pacific J. Math. 197, no. 2 (2001), 291-300. 
[10] M. Golasiński and J. Mukai, Gottlieb and Whitehead center groups of spheres, projective and Moore spaces, Springer, Cham (2014).

[11] D. Gottlieb, A certain subgroup of the fundamental group, Amer. J. Math. 87 (1965), 840-856.

[12] D. Gottlieb, Evaluation subgroups of homotopy groups, Amer. J. Math. 91 (1969), 729-756.

[13] D. Gottlieb, Covering transformations and universal fibrations, Illinois J. Math. 13, no. 2 (1969), 432-437.

[14] H.B. Haslam, $G$-spaces $\bmod F$ and $H$-spaces $\bmod F$, Duke Math. J. 38 (1971), 671-679.

[15] S-T. Hu, Concerning the homotopy groups of the components of the mapping space $Y^{S^{p}}$, Nederl. Akad. Wetensch., Proc. 49 (1946), 1025-1031. Indag. Math. 8 (1946), 623-629.

[16] B. Jahren and S. Kwasik, Free involutions on $\mathbb{S}^{1} \times \mathbb{S}^{n}$, Math. Ann. 351, no. 2 (2011), 281-303.

[17] B-J. Jiang, Estimation of the Nielsen numbers, Acta Math. Sinica 14, 304-312 (in Chinese); translated as Chinese Math. Acta 5 (1964), 330-339.

[18] D.W. Kahn, Induced maps for Postnikov systems, Trans. Amer. Math. Soc. 107, no. 3 (1963), 432-450.

[19] R. Kim and N. Oda, The set of cyclic-element preserving maps, Topology Appl. 160, no. 6 (2013), 794-805.

[20] J. Oprea, Finite group actions on spheres and the Gottlieb group, J. Korean Math. Soc. 28, no. 1 (1991), 65-78.

[21] J. Pak and M.H. Woo, Gottlieb groups on lens spaces, Bull. Korean Math. Soc. 36, no. 3 (1999), 621-627.

[22] C.B. Thomas and C.T.C. Wall, The topological spherical space form problem. I, Compos. Math. 23 (1971), 101-114.

[23] H. Toda, Composition methods in homotopy groups of spheres, Ann. of Math. Stud. 49, Princeton (1962).

[24] S. Waner, Equivariant homotopy theory and Milnor's theorem, Trans. Amer. Math. Soc. 258, no. 2 (1980), 351-368.

[25] G.W. Whitehead, Elements of homotopy theory, Grad. Texts in Math. 61, Springer-Verlag (1978).

Marek Golasiński marekg@matman.uwm.edu.pl

Faculty of Mathematics and Computer Science, University of Warmia and Mazury, Słoneczna 54 Street, 10-710 Olsztyn, Poland

Thiago de Melo tmelo@rc.unesp.br

São Paulo State University (Unesp), Institute of Geosciences and Exact Sciences, Av. 24A, 1515, Bela Vista, CEP 13.506-900, Rio Claro-SP, Brazil 